\title{
Improved Lightning Protection of Carbon Fiber Reinforced Polymer Wind Turbine Blades: Epoxy/Graphene Oxide Nanocomposites
}

\author{
O. Vryonis, T. Andritsch, A. S. Vaughan, P. L. Lewin \\ The Tony Davies High Voltage Laboratory \\ Electronics and Computer Science \\ University of Southampton \\ Southampton, SO17 1BJ, UK \\ O.Vryonis@soton.ac.uk
}

\begin{abstract}
Nanodielectric composites belong to a type of materials engineered for improved performance in several different fields. Advanced technology devices require a new class of materials, combining suitable electrical and thermal properties, as well as, mechanical performance and easy processing. In nanocomposites, a key parameter is the interaction between the matrix and the nanofiller. In the present work, the effect of filler load of graphene oxide (GO), on the thermal and electrical response of epoxy-based nanocomposites was investigated. The final goal of the project is to develop epoxy nanocomposite systems which will meet the requirements to be utilized as a matrix, in carbon fiber reinforced polymer (CFRP) wind turbine blades with improved lightning protection performance. It was found that a filler load of $3 \mathrm{wt} \%$ leads to percolation threshold. The dielectric spectroscopy results confirm the percolation and also show inert dipoles with increasing filler load. The high filler load leads also to lower impulse breakdown strength. The thermal response is affected by the oxygen-based groups of the GO. It was also found that the dispersion/exfoliation method used affects the response of the epoxy resin.
\end{abstract}

\section{INTRODUCTION}

Graphene has attracted the interest of the scientific community over the last decade, since its first isolation in 2004 [1], because of its novel properties. However, expanded graphite has been used as a filler for polymers for over 100 years [2] and, more recently, graphite with a thickness less than $100 \mathrm{~nm}$ was prepared and termed graphite nanoplatelets (GNPs) [3]. Nevertheless, the majority of recent work in this area has been conducted using graphene oxide (GO), which has attracted interest because it is easy to obtain in large amounts, is easier to exfoliate and disperse and its functional groups are available for bonding with the polymer host. Consequently, GO is often preferred to GNPs and pristine graphene [4].

In GO, it is generally accepted that hydroxyl and epoxide groups exist on the basal plane, while carboxyl groups exist on the edges of each sheet [4]. This functionality facilitates the formation of covalent bonds with appropriate matrix polymers, leading to stress transfer from the relatively low modulus matrix to the high modulus filler. Such features, together with parallel improvements in electrical and thermal performance [5] have caused the scientific community to focus on graphene nanocomposites. Indeed, epoxy-based nanocomposites containing GO have been shown to exhibit remarkably enhanced mechanical performance at low filler loadings, which is attributed to the presence of the functional groups discussed above [6]. However, these moieties may also have an impact on crosslinking reactions during curing [7].

The study described here set out to achieve enhancement of the electrical and thermal performance of epoxy systems used as matrices in CFRP based wind turbine blades. In this way the anisotropic behavior of CFRPs, which leads to poor performance during lightning strikes, will be reduced.

\section{EXPERIMENTAL}

\section{A. Materials}

The resin used in this study was DER 332 (Dow), a bisphenol A diglycidyl ether type of epoxy resin, with epoxide percentage of $24.6-25.1 \%$ and a viscosity of $4000-6000 \mathrm{mPa} \cdot \mathrm{s}$ at $25{ }^{\circ} \mathrm{C}$. The hardener used was JEFFAMINE D-230 (Huntsman), a polyetheramine with minimum amine percentage of $97 \%$ and a viscosity of $9.5 \mathrm{mPa} \cdot \mathrm{s}$. The filler used was graphene oxide powder supplied by Sigma Aldrich, which is characterized by a percentage of edge oxidization of $4-10 \%$ and where each grain consists of 15-20 layers,

\section{B. Sample Preparation}

Before any mixing procedure, it was ensured that the epoxy resin had not crystallized and was, therefore, completely transparent. If crystals were visible, it was heated to $55^{\circ} \mathrm{C}$ and then left under ambient conditions to cool down. The mixing procedure started with the GO powder being added to the hardener, in order to take exploit its low viscosity and therefore easy processability. The mixture of GO and hardener was sonicated using an ultrasonic bath (UW DP201-00) for 20 min, in order to disperse and exfoliate the GO. The maximum recorded temperature of the water bath was $33^{\circ} \mathrm{C}$, thereby ensuring that the mixture did not experience a high temperature. The next step was addition of the resin, which was followed by magnetic stirring for $20 \mathrm{~min}$ at ambient temperature. The resin/hardener ratio was 1000:344 and the chosen filler loading levels were $0.1,0.5,1$ and $3 \mathrm{wt} \%$, along with a neat epoxy sample and a reference epoxy sample, as described below. Subsequently, the mixture was sonicated again for $20 \mathrm{~min}$ in 
order further to exfoliate and disperse the GO within the resin. The temperature was again monitored in order to ensure low temperature mixing. After sonication, the mixture was degassed for 20 min a vacuum oven at ambient temperature.

Film sample were prepared using molds comprising of metal plates with a Melinex spacer sandwiched between them, which determines the sample thickness $(<200 \mu \mathrm{m})$. A release agent (QZ13) applied to the mould, prior to assembly. The resin was vacuum cast into the molds, placed inside a fan oven for curing at $80^{\circ} \mathrm{C}$ for $2 \mathrm{~h}$ and then left to cool down slowly to room temperature. Next, the resulting solid samples were taken out of the molds, placed back into the fan oven and post cured at 125 ${ }^{\circ} \mathrm{C}$ for $3 \mathrm{~h}$ and, again, left to cool down slowly. The beneficial effect of post curing treatment on GO/epoxy nanocomposites is discussed elsewhere [8]. Finally, the samples were stored inside a desiccator to prevent uptake of ambient moisture from the environment. The neat epoxy sample was prepared using just 5 min of magnetic stirring with the hardener, followed by degassing and casting into the mould. The reference epoxy sample, despite being unfilled, was prepared using the same procedure as used to prepare the nanocomposites, except that no GO powder was included, in order to investigate the impact of the specific preparation procedure on the epoxy resin itself.

\section{Characterization}

Differential scanning calorimetry (DSC) was used to examine the glass transition, $T_{g}$, in each specimen. A Perkin-Elmer DSC7 system was used, controlled with Pyris software, and was routinely calibrated with high purity indium. All samples had a mass of $\sim 5 \mathrm{mg}$ and were sealed in aluminum cans. The temperature profile involved isothermal heating at $40{ }^{\circ} \mathrm{C}$ for 1 min, following by ramping to $160{ }^{\circ} \mathrm{C}$ at a heating rate of 10 $\mathrm{K} / \mathrm{min}$. The resulting thermographs were normalized with respect to the sample's mass and then the $T_{g}$ was determined from the point of inflection of the step like transition of the heat flow (Table I).

Thermogravimetric analysis (TGA) was used to study the thermal stability of the samples. The system (Perkin-Elmer Pyris 1 TGA) was also controlled using Pyris software. All samples had a mass $>2 \mathrm{mg}$, were placed in an aluminum can and underwent isothermal heating at $50{ }^{\circ} \mathrm{C}$ for $1 \mathrm{~min}$, followed by a $10 \mathrm{~K} / \mathrm{min}$ ramp up to $600{ }^{\circ} \mathrm{C}$. All measurements were performed in an oxygen environment.

DC conductivity was measured using a capacitor like arrangement with circular gold coated electrodes and a guard ring configuration, to reduce fringing effects at the edge of the sample, placed inside an oven. The system using in-house software and samples were sputter coated with a $20 \mathrm{~mm}$ diameter contacts on their upper and lower surfaces, to improve contact with the measuring system. All measurements were conducted at ambient temperature and at a constant electric field of $40 \mathrm{kV} / \mathrm{mm}$ for $3 \mathrm{~h}$. A ramp up to the desired electric field was used with a starting voltage of $100 \mathrm{~V}$, a voltage step of $100 \mathrm{~V}$ and a time step of $10 \mathrm{sec}$.

Impulse breakdown strength measurements were made by following the BS EN 60243-3:2013 standard, which specifies a $1.2 / 50 \mu$ s lightening impulse waveform. These tests were conducted at room temperature, using a sphere/sphere arrangement with $6.3 \mathrm{~mm}$ diameter ball bearing electrodes, allowing easy replacement before surface damage could affect the obtained data. The exact specimen thickness was measured at each breakdown point in order to convert the measured breakdown voltage into a breakdown field. The sample and electrodes were fully immersed in silicone oil in order to avoid flashovers.

The dielectric response of the samples was measured via broadband dielectric spectroscopy (BDS), using a Solartron 1296 dielectric interface in combination with a Schlumberger SI 1260 impedance/phase gain analyser and a Lake Shore 332 temperature controller. The sample was mounted on a Janis Research STVP-200-XG system cryostat, which was capable of performing electrical measurements over the temperature range of $\sim 5-600 \mathrm{~K}$. The system included an integrated variable temperature sample chamber, a vacuum insulated cryostat body, a sample positioner assembly with sample holder, all interconnected with the temperature controller. Liquid nitrogen was used to cool the sample. The temperature range used for this study was -100 to $80{ }^{\circ} \mathrm{C}$.

\section{RESULTS}

Table I presents DSC $T_{g}$ values for all samples, which are considered reasonable for this epoxy resin [8]. It can be seen that the incorporation of GO powder inside the epoxy resin does not provide any significant alteration in the $T_{g}$ of the system. All samples exhibit a $T_{g}$ of $87 \pm 2{ }^{\circ} \mathrm{C}$; the variations seen Table I fall within the experimental uncertainty.

TABLE I

FILLER LOADINGS AND THE RESPECTIVE GLASS TRANSITION TEMPERATURES

\begin{tabular}{|l|c|c|}
\hline \multicolumn{1}{|c|}{ sample } & GO vol\% & $T_{g}\left({ }^{\circ} \mathrm{C}\right)$ \\
\hline Neat epoxy & 0 & 87.5 \\
\hline Reference epoxy & 0 & 87.9 \\
\hline $0.1 \mathrm{wt} \% \mathrm{GO}$ & 0.065 & 87.0 \\
\hline $0.5 \mathrm{wt} \% \mathrm{GO}$ & 0.325 & 85.1 \\
\hline $1 \mathrm{wt} \% \mathrm{GO}$ & 0.65 & 87.2 \\
\hline $3 \mathrm{wt} \% \mathrm{GO}$ & 1.95 & 89.3 \\
\hline
\end{tabular}

Fig. 1 contains TGA data obtained from all the material systems considered here. All formulations display comparable thermal stabilities and do not exhibit any significant mass loss up to $\sim 250{ }^{\circ} \mathrm{C}$. Nevertheless, it is obvious that the reference epoxy resin is more thermally stable than every other sample, including the neat epoxy sample. It is worth noting that the mass of the residue at $600{ }^{\circ} \mathrm{C}$ decreases as the loading of $\mathrm{GO}$ increases.

Fig. 2 illustrates the DC conductivity of all the samples at room temperature, from which it is evident that no significant variations occur for filler loadings up to $0.5 \mathrm{wt} \%$. However, the sample containing $1 \mathrm{wt} \%$ of GO exhibits a higher conductivity by one order of magnitude, while a further increase in filler loading to $3 \mathrm{wt} \%$ results in six orders of magnitude increase in conductivity. It is worth noting that the neat epoxy and the reference epoxy give comparable values.

Impulse withstand field data are shown in Fig. 3, where the opposite behavior, compared to conductivity, is evident. While 
the samples up to $1 \mathrm{wt} \%$ GO exhibit similar values of withstand fields, the $3 \mathrm{wt} \%$ sample displayed one order of magnitude lower. The highest withstand field recorded was for the reference epoxy sample.

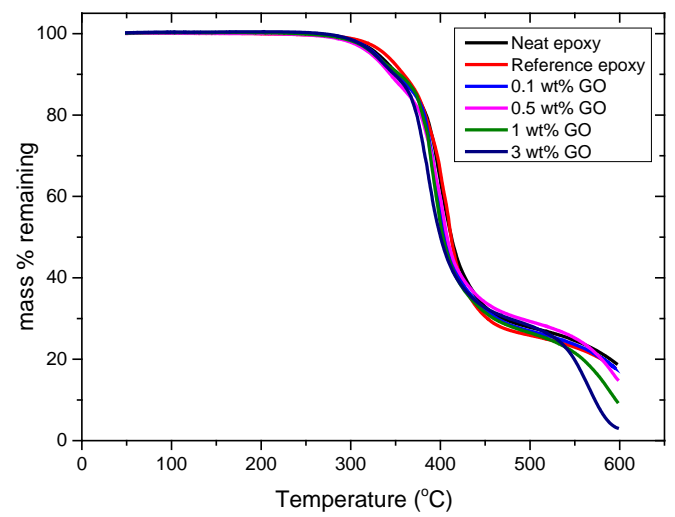

Fig. 1. TGA plot for all samples, heated in air at $10^{\circ} \mathrm{C} / \mathrm{min}$

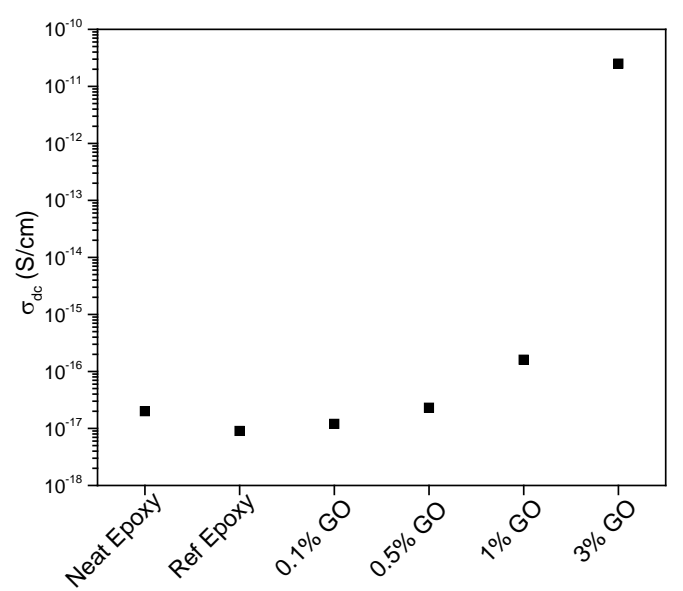

Fig. 2. DC conductivity of all the samples at room temperature.

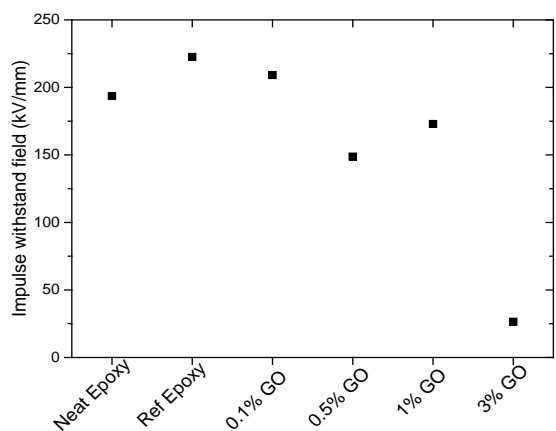

Fig. 3. Impulse withstand fields for all samples with waveform 1.2/50 $\mu \mathrm{s}$

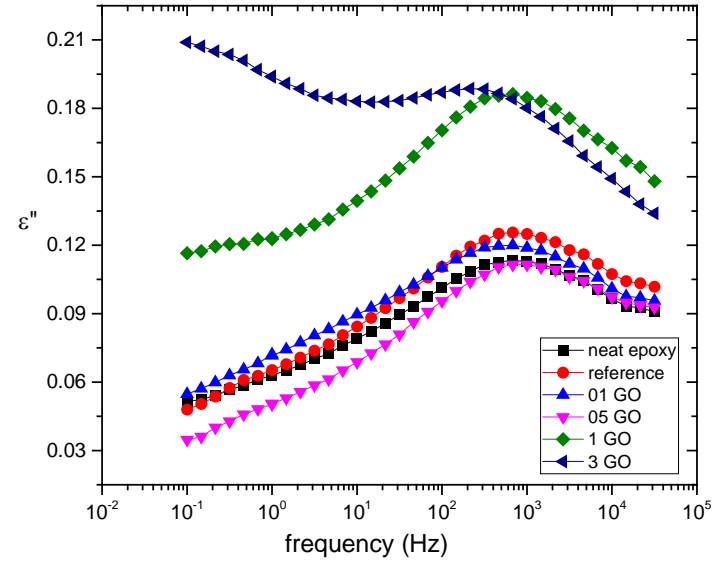

Fig. 4. Imaginary part of relative permittivity versus frequency at $-20^{\circ} \mathrm{C}$.

Finally, the imaginary part of the relative permittivity as a function of frequency at $-20^{\circ} \mathrm{C}$ is illustrated at Fig. 4. It is clear from this that the 1 and $3 \mathrm{wt} \%$ samples exhibit higher dielectric losses than the other samples. A pronounced $\beta$ - relaxation peak is revealed for all samples, which is associated with motions of the hydroxyl-ether groups on the backbone that result from crosslinking reactions between the epoxy and the hardener. The peak seems to be shifted to lower frequency for $3 \mathrm{wt} \%$ filler load. It is important to note that another mechanism can be seen in the $3 \mathrm{wt} \%$ sample, at lower frequency, which nearly overlaps the $\beta$ - relaxation peak. This peak-like process is temperature independent and is associated with the high conductivity of this sample.

\section{DISCUSSION}

Fig. 5 presents the variation in the position of $\beta$ - relaxation loss peak as a function of frequency and temperature. The limited number of data points plotted for the specimen containing 3 wt $\%$ of GO result from overlap with the conductivity-related peak, so preventing the detection of more

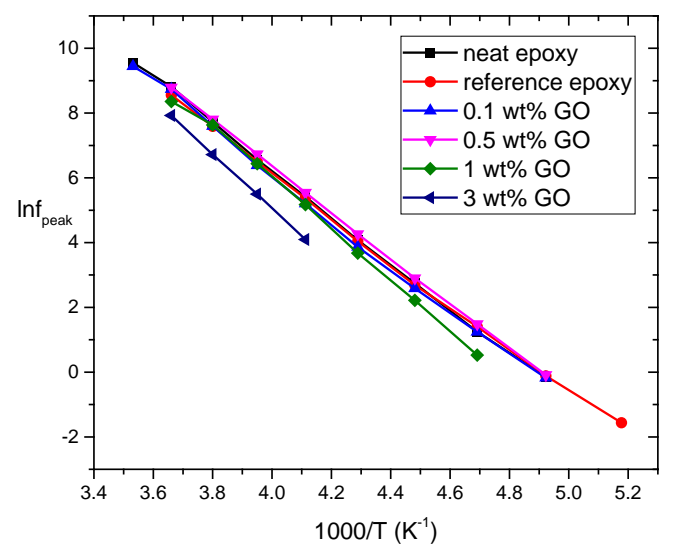

Fig. 5. Loss peak position ( $\beta$ - relaxation) as a function of frequency and temperature. 
$\beta$ - relaxation peaks. Nevertheless, the data acquired from all six systems are consistent and reveal a good linear dependence when plotted as shown. Thus, this relaxation process conforms to an Arrhenius- type behaviour, whereby:

$$
f_{\text {peak }}=f_{0} e^{-\frac{E_{A}}{k_{B} T}}
$$

where, $f_{0}$ is a pre-exponential factor, $E_{A}$ is the activation energy, $T$ is the absolute temperature and $k_{B}$ is the Boltzmann constant. The corresponding activation energies are presented in Table II, from which it is evident that a higher filler load leads to higher activation energy. Furthermore, it is confirmed that $\beta$ relaxation is displaced to a lower frequency, at a fixed temperature, for the $3 \mathrm{wt} \%$ sample, which means that the associated molecular mechanism is retarded, requiring more time, or energy through heating, to align with the applied field. This is also confirmed by the high activation energy value obtained from this sample. It is also worth mentioning that the preparation procedure has an impact on the epoxy resin itself, as the reference epoxy displays lower activation energy than the neat epoxy sample. Taking into account the percolation threshold revealed for this sample, it can be assumed that the conductive path formed between the GO layers, results in limited space for the dipole movement, thus, requiring more energy. The percolation can also be considered as poor dispersion as it also leads to lower impulse breakdown strength. The higher conductivity is also evinced by the pronounced, temperature-independent, loss peak at low frequencies in the dielectric spectra. The $T_{g}$ independence on the filler load can be ascribed to the low oxidation levels of this filler, as only edge oxidation (carboxyl groups) is mentioned, which means there are no epoxide groups, on the basal plane to react with the hardener. Even this amount of oxygen-based groups leads to a reduction in the thermal stability of the nanocomposites, as evinced by their relatively fast decomposition, even though the preparation method makes the host polymer (reference epoxy) more thermally stable.

It can be assumed that a finer quality of filler, with excess functional groups, such as epoxides, could lead to different bonding with the matrix and thus to dramatic changes of the overall behaviour described above.

TABLE II

ACTIVATION ENERGIES AS RECEIVED FROM ARRHENIUS TYPE PLOTS

\begin{tabular}{|l|c|}
\hline \multicolumn{1}{|c|}{ sample } & $E_{A}(\mathrm{eV})$ \\
\hline Neat epoxy & $0.61 \pm 0.01$ \\
\hline Reference epoxy & $0.58 \pm 0.01$ \\
\hline $0.1 \mathrm{wt} \% \mathrm{GO}$ & $0.61 \pm 0.01$ \\
\hline $0.5 \mathrm{wt} \% \mathrm{GO}$ & $0.61 \pm 0.003$ \\
\hline $1 \mathrm{wt} \% \mathrm{GO}$ & $0.67 \pm 0.01$ \\
\hline $3 \mathrm{wt} \% \mathrm{GO}$ & $0.73 \pm 0.01$ \\
\hline
\end{tabular}

\section{CONCLUSIONS}

The effect of filler load of graphene oxide inside an epoxy resin matrix has been investigated via electrical and thermal characterization. The alteration of the preparation procedure, in order to exfoliate and disperse the GO, made it necessary to investigate the impact on the epoxy resin itself. The glass transition did not show any alterations, which is ascribed to the absence of excess epoxide groups on the GO surface, though the presence of carboxyl groups reduced the thermal stability of the system. It was also found that the preparation procedure improved the thermal stability of the epoxy resin. A percolation threshold was revealed at $3 \mathrm{wt} \%$, leading to an increase in electrical conductivity of six orders of magnitude; this results from bridging between GO layers. The percolated sample exhibited reduced breakdown strength by one order of magnitude and a pronounced, temperature independent, conductivity-related loss peak in the low frequency regime of the dielectric spectra. The dynamic molecular analysis indicated a progressive obstruction of dipole movement with increasing filler load, possibly due to space limitations inside the matrix, as well as an effect of the preparation procedure on the dipole mobility. A filler with alternative functional groups could lead to different performance.

The most important goal, the increase of electrical conductivity of the resin was achieved, which can potentially lead to quicker electrical current dissipation during a lightning strike. Also, as the difference of the electrical conductivity of the CFRP constituent material reduces, the risk of flashovers leading to mechanical damage is reduced. Knowledge about the dielectric response and the dipole movement is also very important as it affects the flow of current inside the material. Slight alterations of the resin's $T_{g}$ is also an important parameter for this application, in order to prevent damage and consequently material failure.

\section{ACKNOWLEDGMENTS}

This work was sponsored by Marie Sklodowska Curie Actions, Innovative Training Networks (ITN), Call: H2020MSCA-ITN-2014.

The authors would like to thank Dr. Suvi Virtanen, and Dr. Matt Praeger for their useful help throughout the study.

\section{REFERENCES}

[1] K. S. Novoselov, et al. "Electric field effect in atomically thin carbon films." Science Vol. 306, Issue 5696, pp.666-669, 2004.

[2] A. Celzard, J. F. Mareche, and G. Furdin. "Surface area of compressed expanded graphite." Carbon Vol. 40, Issue 14, pp.2713-2718, 2002.

[3] B. Z. Jang, and A. Zhamu. "Processing of nanographene platelets (NGPs) and NGP nanocomposites: a review." Journal of Materials Science Vol. 43 Issue 15, pp.5092-5101, 2008.

[4] R. J. Young, Kinloch I. A., Gong, L., and K. S. Novoselov, "The mechanics of graphene nanocomposites: a review.” Composites Science and Technology, Vol.72 Issue 12, pp.1459-1476, 2012.

[5] J. R. Potts, D. R. Dreyer, C. W. Bielawski, and R. S. Ruoff. "Graphenebased polymer nanocomposites.” Polymer, Vol. 52, Issue 1, pp.5-25 2011.

[6] Z. Li, et al. "The role of functional groups on graphene oxide in epoxy nanocomposites." Polymer Vol.54 Issue 21, pp.5821-5829, 2013.

[7] D. Galpaya, et al. "Fabrication and characterisation of graphene oxideepoxy nanocomposite." Fourth International Conference on Smart Materials and Nanotechnology in Engineering. International Society for Optics and Photonics, August 2013.

[8] P. Mancinelli, et al. "Preparation and dielectric behavior of epoxy resin containing graphene oxide." Solid Dielectrics (ICSD), 2013 IEEE International Conference, June 2013. 